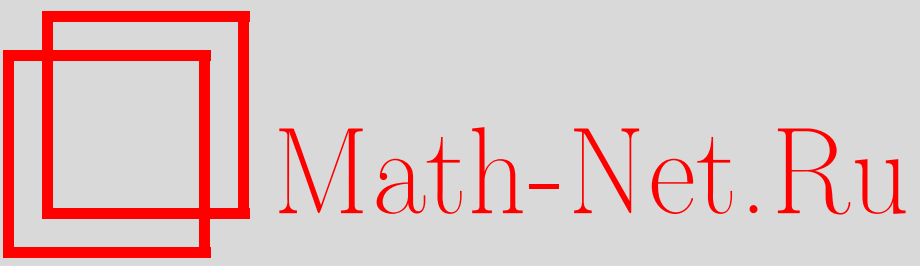

Ю. Т. Лисица, О (ко)гомологически локально связных пространствах, $У М H$, 2003, том 58, выпуск 6, 153-154

DOI: https://doi.org/10.4213/rm683

Использование Общероссийского математического портала Math-Net.Ru подразумевает, что вы прочитали и согласны с пользовательским соглашением

http://www . mathnet.ru/rus/agreement

Параметры загрузки:

IP : 54.224 .60 .19

26 апреля 2023 г., 13:39:09 


\title{
О (КО)ГОМОЛОГИЧЕСКИ ЛОКАЛЬНО СВЯЗНЫХ ПРОСТРАНСТВАХ
}

\author{
Ю. Т. ЛисицА
}

Автором в [1] были введены сильные пограничные гомологические группы $\bar{H}_{m}^{b r}(X ; G)=$ $\lim _{\rightarrow \mu} \bar{H}_{m}\left(X, K^{\mu} ; G\right)$ и сильые пограничные когомологические группы $\bar{H}_{b r}^{m}(X ; G)=$ $H^{m}\left(\operatorname{holim}_{\leftarrow \mu} C^{*}\left(X, K^{\mu} ; G\right)\right)$ вполне регулярного топологического пространства $X$ с коэффициентами в абелевой группе $G$. Здесь $K^{\mu} \subseteq X, \mu \in M,-$ все компактные подмножества в $X$, упорядоченные по вложению, $\bar{H}_{m}\left(X, K^{\mu} ; G\right) \stackrel{\text { def }}{=} H_{m}\left(\operatorname{holim}_{\leftarrow \lambda} C_{*}\left(\underline{X}, \underline{K}^{\mu} ; G\right)\right)$ - сильные относительные гомологические группы (определенные с помощью $A N R$-резолюции Мардешича $\underline{p}=\left(p_{\lambda}\right):\left(X, K^{\mu}\right) \rightarrow\left(\underline{X}, \underline{K}^{\mu}\right)=\left(\left(X_{\lambda}, K_{\lambda}^{\mu}\right), p_{\lambda \lambda^{\prime}}, \Lambda\right), X_{\lambda}, K_{\lambda}^{\mu} \in A N R,[2]$ и соответственно обратной системы сингулярных комплексов $C_{*}\left(X_{\lambda}, K_{\lambda}^{\mu} ; G\right)$, а $C^{*}\left(X, K^{\mu} ; G\right)$ - относителшные коцепные комплексы Александера-Спеньера и holim $\leftarrow$ - функтор гомотопического обратного предела. Эти группы связывают окончательным образом классические гомологические группы с компактными носителями $\bar{H}_{m}^{c}(X ; G) \stackrel{\text { def }}{=} \lim _{\rightarrow \mu} \bar{H}_{m}\left(K^{\mu} ; G\right)$ и сильные гомологические группы $\bar{H}_{m}(X ; G) \stackrel{\text { def }}{=} H_{m}\left(\operatorname{holim}_{\leftarrow \lambda} C_{*}\left(X_{\lambda} ; G\right)\right)$, с помощью следующей длинной точной последовательности:

$$
\cdots \rightarrow \bar{H}_{m}^{c}(X ; G) \stackrel{\bar{\mu}_{m}}{\rightarrow} \bar{H}_{m}(X ; G) \rightarrow \bar{H}_{m}^{b r}(X ; G) \rightarrow \bar{H}_{m-1}^{c}(X ; G) \rightarrow \cdots,
$$

и соответственно когомологические группы Александера-Спеньера $H^{m}(X ; G)$ и сильные когомологические группы $\bar{H}^{m}(X ; G) \stackrel{\text { def }}{=} H^{m}\left(\operatorname{holim}_{\leftarrow \mu} C^{*}\left(K^{\mu} ; G\right)\right)$, введенными автором [6], в виде длинной точной последовательности:

$$
\cdots \rightarrow \bar{H}^{m-1}(X ; G) \rightarrow \bar{H}_{b r}^{m}(X ; G) \rightarrow H^{m}(X ; G) \stackrel{\bar{\lambda}^{m}}{\rightarrow} \bar{H}^{m}(X ; G) \rightarrow \cdots
$$

Спрашивается: когда естественные гомоморфизмы $\bar{\mu}_{m}: \bar{H}_{m}^{c}(X ; G) \rightarrow \bar{H}_{m}(X ; G)$ и $\bar{\lambda}^{m}$ : $H^{m}(X ; G) \rightarrow \bar{H}^{m}(X ; G)$ являются изоморфизмами для любого целого $m$ ? Что эквивалентно вопросу о тривиальности сильных пограничных (ко)гомологических групп.

Ответ на этот вопрос для сильных пограничных (ко)гомологий дают следующие теоремы.

Теорема 1. Если для каждого $\mu \in M$ существует такое $\mu^{\prime} \geqslant \mu$, что $i_{m}^{\mu^{\prime}}: \bar{H}_{m}\left(K^{\mu^{\prime}} ; G\right) \rightarrow \bar{H}_{m}(X ; G)$ является эпиморфизмом, $\quad$ а $\operatorname{Ker}\left(i_{m}^{\mu}: \bar{H}_{m}\left(K^{\mu} ; G\right) \rightarrow\right.$ $\left.\bar{H}_{m}(X ; G)\right)=\operatorname{Ker}\left(i_{m}^{\mu \mu^{\prime}}: \bar{H}_{m}\left(K^{\mu} ; G\right) \rightarrow \bar{H}_{m}\left(K^{\mu^{\prime}} ; G\right)\right)$, то $\bar{\mu}_{m}-$ изоморфизм. Если в дополнение $\kappa$ әтому $i_{m+1}^{\mu^{\prime}}: \bar{H}_{m+1}\left(K^{\mu^{\prime}} ; G\right) \rightarrow \bar{H}_{m+1}(X ; G)$ является әпиморфизмом, mo $\bar{H}_{m+1}^{b r}(X ; G)=0$.

Tеорема 2. Если для каждого $\lambda \in \Lambda$ существ ует такое $\lambda^{\prime} \geqslant \lambda$, ито $i_{\lambda^{\prime} n}: \bar{H}_{n}^{c}(X ; G)$ $\rightarrow H_{n}\left(X_{\lambda^{\prime}} ; G\right)$ является мономорфизмом для любого $n \geqslant m-1, a \operatorname{Im}\left(i_{\lambda n}: \bar{H}_{n}^{c}(X ; G) \rightarrow\right.$ $\left.H_{n}\left(X_{\lambda} ; G\right)\right)=\operatorname{Im}\left(p_{\lambda \lambda^{\prime} n}: \bar{H}_{n}^{c}\left(X_{\lambda^{\prime}} ; G\right) \rightarrow H_{n}\left(X_{\lambda} ; G\right)\right)$ для всех $n \geqslant m$, mо $\bar{H}_{n}^{b r}(X ; G)=$ 0 для всех $n \geqslant m$ u, следовательно, $\bar{\mu}_{n}$ является изоморфизмом для всех $n \geqslant m u$ мономорфизмом для $n=m-1$. Более того, $\bar{H}_{n}(X ; G) \approx \check{H}_{n}(X ; G)$ для всех $n \geqslant m$.

ТеОрема 3. Пусть $X$ - паракомпактное хаусдорфово пространство, являющееся гомологически локально связным в любой размерности по отношению $\kappa$ классическим гомологиям с компактными носителями, т.е. $X \in h c_{G}^{\infty}$. Тогда $\bar{H}_{m}^{b r}(X ; G)=0$ для любого челого $m$ u, следовательно, $\bar{\mu}_{m}$ является изоморфизмом для любого $\mathrm{m}$.

Работа выполнена при поддержке Российского фонда фундаментальных исследований (грант № 00-01-00304). 
Теорема 4. Если для каждого $\mu \in M$ существует такое $\mu^{\prime} \geqslant \mu$, ито $i^{\mu^{\prime} n}: H^{n}(X ; G)$ $\rightarrow H^{n}\left(K^{\mu^{\prime}} ; G\right)$ является мономорфизмом для всех $n \leqslant m+1 u \operatorname{Im}\left(i^{\mu n}: H^{n}(X ; G) \rightarrow\right.$ $\left.H^{n}\left(K^{\mu} ; G\right)\right)=\operatorname{Im}\left(i^{\mu \mu^{\prime} n}: H^{n}\left(K^{\mu^{\prime}} ; G\right) \rightarrow H^{n}\left(K^{\mu} ; G\right)\right)$ для всех $n \leqslant m$, mо $\bar{H}_{b r}^{n}(X ; G)=0$ для всех $n \leqslant m+1$ и, следовательно, $\bar{\lambda}^{n}-$ изоморфизм для всех $n \leqslant m$ и мономорфизм для $n=m+1$. Более того, $\bar{H}^{n}(X ; G) \approx \lim _{\leftarrow \mu} H^{n}\left(K^{\mu} ; G\right)$.

Теорема 5. Если для каждого $\lambda \in \Lambda$ существует такое $\lambda^{\prime} \geqslant \lambda$, ито $i_{\lambda^{\prime}}^{m}: H^{m}\left(X_{\lambda} ; G\right)$ $\rightarrow \bar{H}^{m}(X ; G)$ является эпиморфизмом, $\quad$ а $\operatorname{Ker}\left(p_{\lambda \lambda^{\prime}}^{m}: H^{m}\left(X_{\lambda} ; G\right) \rightarrow H^{m}\left(X_{\lambda^{\prime}} ; G\right)\right)=$ $\operatorname{Ker}\left(i_{\lambda}^{m}: H^{m}\left(X_{\lambda} ; G\right) \rightarrow \bar{H}^{m}(X ; G)\right)$, mо $\bar{\lambda}^{m}-$ изоморфизм. Если в дополнение $к$ этому $i_{\lambda^{\prime}}^{m-1}$ является әпимор физмом, то $\bar{H}_{b r}^{m}(X ; G)=0$.

Теорема 6. Пусть $X$ - паракомпактное хаусдорфово пространство, являющееся когомологически локально связным в размерности $m$ по отношению $\kappa$ сильным когомологиям, m.e. $X \in \operatorname{sclc}_{G}^{m}$. Тогда $\bar{H}_{b r}^{n}(X ; G)=0$ для всех $n \leqslant m$ и, следовательно, $\bar{\lambda}^{n}$ изоморфизм для всех $n \leqslant m$ и мономорфизм для $n=m+1$.

Непосредственным следствием части этой теории, касающейся гомологически и когомологически локалњно связных пространств, являются следующие теоремы, представляющие самостоятельный интерес.

Tеорема 7. Пусть $\underline{p}=\left(p_{\lambda}\right): X \rightarrow \underline{X}=\left(X_{\lambda}, p_{\lambda \lambda^{\prime}}, \Lambda\right)$ - резолюция Мардешича пространства $X$ и $X_{\lambda}$ - паракомпактнье хаусдорфовь пространства, являющиеся гомологически локально связными во всех размерностях по отношению $к$ сингулярным гомологиям и гомологиям с компактными носителями, m.е. $X_{\lambda} \in H L C_{G}^{\infty} \cap h l c_{G}^{\infty}, \lambda \in \Lambda$. Тогда соответствующий сингулярный про-цепной комплекс $C_{*}(\underline{X} ; G)$ естественным образом определяет сильные гомологические группы $\bar{H}_{m}(X ; G)$ для любого челого $\mathrm{m}$.

Теорема 8. Пусть $(X, A)$ - замкнутая пара наследственно паракомпактных хаусдорфовых пространств, являющихся (ко) гомологически локально связными в размерности $m$ по отношению $к$ гомологиям с компактными носителями, сильными когомологиями и чеховскими когомологиями соответственно, m.e. $X, A \in h l c^{m}, X, A \in s c l c^{m}$ и $X, A \in$ clc $^{m}$. Тогда фактор-пространство $X / A$ также является (ко)гомологически локально связным в размерности т соответственно, m.e. $X / A \in h l c^{m}, X / A \in$ sclc $^{m}$ и $X / A \in$ clc $^{m}$ соответственно.

Существуют примеры пространств, показьвающие, что, вообще говоря, сильные пограничные (ко)гомологии могут быть нетривиальны [4; с. 247-257]. Доказательство теоремы 1 простое, в отличие от доказательства теоремы 2 , которое требует нетривиального построения ANR-pезолюции Мардешича пространства $X$ с подсистемами из ANR-резолюций для каждого компакта $K^{\mu}$ в $X$. Доказательства теорем 3 и 6 весьма длинные и требуют привлечения спектральной последовательности покрытия как для гомологий, так и для когомологий [5], аддиционной теоремы для сильных когомологий и теоремы типа Брауна-Адамса о представимости сильных когомологий [3], а также многочисленных резултатов из теории гомотопических обратных пределов и резольвент Мардешича.

\section{СПИСОК ЛИТЕРАТУРЫ}

[1] Ju. T. Lisica. Strong border homology and cohomology // International Conference on Geometric Topology II, Dubrovnik, 2002. [2] S. Mardešić // Tsukuba J. Math. 1984. V. 8. № 1. P. 81-93. [3] Ju. T. Lisica. Strong shape with compact supports and strong cohomology // International Conference on Topology and its Applications, Ohrid, 2000. [4] Ю. Т. Лисица. Когерентные гомотопии, гомологии, когомологии и сильная теория шейпов // Дисс. ... докт. физ.-матем. наук. М.: МГУ, 2001. [5] C. N. Lee // Michigan Math. J. 1967. V. 14. P. 207-217. [6] Yu. T. Lisica // Lecture Notes in Math. 1987. V. 1283. P. 148-163. 